\title{
Efecto de la vacunación de cerdas y lechones contra circovirus porcino tipo 2 (PCV2) en la ganancia de peso y porcentaje de mortalidad en México
}

\author{
Effect of vaccination of sow and piglets against porcine circovirus type 2 (PCV2) \\ on weight gain and percentage mortality in Mexico \\ A Villa-Mancera ${ }^{*}$, R Huerta-Crispína , A Córdova-Izquierdob, \\ S Ortega-Vargas ${ }^{\mathrm{a}}$, A Trejo-Córdova ${ }^{\mathrm{c}}$ \\ ${ }^{\text {a }}$ Laboratorio de Genética y Reproducción, Facultad de Medicina Veterinaria y Zootecnia, \\ Benemérita Universidad Autónoma de Puebla, Puebla, México. \\ b Departamento de Producción Agrícola y Animal, Universidad Autónoma Metropolitana, Xochimilco, México. \\ División Académica de Ciencias Agropecuarias, Universidad Juárez Autónoma de Tabasco, Tabasco, México. \\ ${ }^{\mathrm{c}}$ Universidad del Papaloapan, Oaxaca, México.
}

\begin{abstract}
SUMMARY
The aim of this study was to evaluate the efficacy of three different vaccination protocols (sows, piglets and sows and piglets) on average daily weight gain and mortality rate in piglets from farrow to finish farm with postweaning multisystemic wasting syndrome (PMWS). During the period after vaccination (weeks 3-22) the piglets, sows and piglets, exhibited significantly improved average daily weight gain and significantly reduced mortality rate. Compared with the control group, sows and piglets vaccinated against PCV2 had a significantly higher average daily gain and significantly lower mortality rate for the study period (weeks 1-22). It is concluded that vaccination against PCV2 was effective at improving growth performance and reducing mortality rate in a herd infected with PCV2.
\end{abstract}

Key words: PCV2, vaccination protocols, weight gain, mortality.

RESUMEN

El objetivo del presente estudio fue evaluar la eficiencia de tres protocolos de vacunación (cerdas, lechones o cerdas y lechones), sobre la media de la ganancia diaria de peso y porcentaje de mortalidad en lechones desde el parto hasta la finalización, en una granja con Síndrome Multisistémico de Desmedro Postdestete (PMWS). Durante el periodo posterior a la vacunación (semana 3-22) de lechones, cerdas y lechones, éstos mostraron un aumento significativo en la media de la ganancia diaria de peso y una reducción significativa en el porcentaje de mortalidad. Las cerdas y lechones vacunados contra PCV2 para el periodo de estudio (semana 1-22), tuvieron un aumento significativo en la ganancia diaria de peso y una reducción significativa en el porcentaje de mortalidad cuando se les comparó con el grupo control. Estos datos demuestran que la vacunación contra PCV2 fue efectiva en mejorar el crecimiento y reducir el porcentaje de mortalidad en una piara infectada con PCV2.

Palabras clave: PCV2, protocolos de vacunación, ganancia de peso, mortalidad.

\section{INTRODUCCIÓN}

El circovirus porcino tipo 2 (PCV2) es el agente causal del Síndrome Multisistémico de Desmedro Postdestete (PMWS), los principales signos clínicos son retraso en el crecimiento, frecuentemente acompañado de palidez, disnea y en menor medida de diarrea y/o ictericia, principalmente en cerdos de entre 2 y 4 meses de edad, etapa tardía del destete y temprana de engorda (Harding 1998, Segalés y Domingo 2002, Harding 2004). Suele afectar entre

Aceptado: 29.11.2012.

* Proyecto VIEP-BUAP 2011-2013 (VIMA-NAT11-I)

* Laboratorio de Genética y Reproducción, Facultad de Medicina Veterinaria y Zootecnia, Benemérita Universidad Autónoma de Puebla, 4 Sur 304 Col. Centro, CP 75482, Tecamachalco Puebla, México; abel.villa@gmail.com
4-30\% de los animales, de los cuales mueren de 70-80\%, causando importantes pérdidas económicas a la industria porcina (Segalés y Domingo 2002). Otra manifestación clínica de animales infectados con PCV2 son las fallas reproductivas, ocasionando abortos durante la mitad y final de la gestación o lechones nacidos muertos, los cuales muestran miocarditis necrotizante y presencia de PCV2 en tejido cardíaco (West y col 1999, Mikami y col 2005).

Los anticuerpos maternales están presentes en prácticamente todos los lechones tras la toma de calostro y van disminuyendo progresivamente durante la lactación y la transición (Rodríguez-Arrioja y col 2002). La viremia por PCV2 suele aparecer entre la fase final de transición y el inicio de la fase de engorda, coincidiendo con el momento en el que los anticuerpos maternales alcanzan niveles mínimos (Sibila y col 2004). Se ha descrito que 
un bajo porcentaje de animales pueden presentar ya la infección por PCV2 a los pocos días de vida (Sibila y col 2004, Calsamiglia y col 2007).

Las vacunas comerciales contra PCV2 disponibles en el mercado difieren en el tipo de antígeno y adyuvante, animales (cerdas y/o lechones) y dosis (una o dos) (Chae 2012). La vacuna comercial Circovac ${ }^{\circledR}$ (Merial) introducida en 2006, está compuesta por virus inactivado y contiene aceite de parafina ligero como adyuvante; inicialmente fue utilizada sólo en cerdas $(2 \mathrm{ml})$ y posteriormente aplicada a lechones $(0,5 \mathrm{ml})$ (Fraile y col 2012). El principio de la vacunación de cerdas es conferir inmunidad pasiva para proteger a los lechones recién nacidos contra la exposición a PCV2, mientras estos son altamente susceptibles; en contraste, la vacunación de lechones induce inmunidad activa contra enfermedades asociadas a circovirus porcino (PCVD) (Opriessnig y col 2010, Beach y Meng 2012, Chae 2012).

Las vacunas comerciales actualmente disponibles contra PCV2 son herramientas efectivas en el control del PMWS en condiciones de campo y experimentales. La vacunación de cerdas primerizas y adultas bajo condiciones de campo, incrementa los títulos de anticuerpos en suero, reduce la carga viral en leche y calostro, incrementando la ganancia diaria de peso (GDP) y reduciendo los porcentajes de mortalidad de su descendencia (Pejsak y col 2010, Gerber y col 2011, Kurmann y col 2011, Fraile y col 2012). La vacunación de lechones contra PCV2, induce una respuesta de anticuerpos neutralizantes, una respuesta inmune humoral mediada por células secretoras de IFN- $\gamma$, reduciendo o retrasando la infección por PCV2 durante el destete o engorda (Fort y col 2008, Kekarainen y col 2010, Opriessnig y col 2010). El objetivo de este estudio fue evaluar el efecto de tres protocolos de vacunación contra PCV2 en cerdas y lechones sobre la ganancia de peso y porcentaje de mortalidad.

\section{MATERIAL Y MÉTODOS}

\section{LOCALIZACIÓN}

La unidad de producción porcina se ubica en el estado de Puebla, México; es una granja porcina con un sistema de producción en tres sitios, con flujo todo dentro/todo fuera por edificio. En el sitio uno se encuentra el área de gestación y maternidad (etapa de lactancia: semana 1-3), en el sitio dos se tienen a los cerdos destetados (semana 3-10) y en el sitio tres a los animales en etapa de engorda o finalización (semana 10-22). Esta unidad productiva tiene un inventario de 4.500 hembras y produce alrededor de 2.000 cerdos semanales.

Como antecedente, la granja fue diagnosticada con PCVD asociado con PCV2, confirmado en base a los signos clínicos (palidez, desmedro, diarrea y tos) y carga viral por PCR cuantitativa. En la necropsia, los animales revelan evidencia de síndrome de dermatitis y nefropatía porcina con lesiones en piel de color rojo o púrpura, PMWS con depleción linfoidea, en los pulmones se observan vasculitis y neumonía intersticial. La granja es seropositiva pero estable a PRRS (Herdcheck2XR, IDEXX; Civtest Suis PRRS A/S, Hipra; Civtest Suis PRRS E/S, Hipra) negativa al virus de influenza porcina (Civtest Suis Influenza, Hipra) y cepas patógenas de Actinobacillus pleuropneumoniae (APP-ApxIV, IDEXX). Los cerdos fueron vacunados a los 7 y 21 días de edad contra Haemophilus parasuis y Mycoplasma hyopneumoniae a los 23 días de edad.

\section{DISEÑO EXPERIMENTAL}

Se seleccionaron 76 cerdas para ser dividas en cuatro grupos, de acuerdo a su paridad. Se utilizaron 3 diferentes protocolos de vacunación contra PCV2: 1) cerdas vacunadas, lechones no vacunados, 2) cerdas no vacunadas, lechones vacunados, 3) cerdas vacunadas, lechones vacunados; grupo control: cerdas no vacunadas, lechones no vacunados (cuadro 1). Las cerdas fueron vacunadas en dos ocasiones con un intervalo de 3-4 semanas antes del parto, mientras que los lechones fueron inmunizados a las 3 semanas de edad con 0,5 ml Circovac ${ }^{\circledR}$ (Merial). Los lechones fueron identificados individualmente en la oreja para su monitoreo y evaluación. Los animales del grupo control o sin vacunar fueron inyectados con agua estéril.

El porcentaje de mortalidad para cada grupo de cerdos vacunados y etapa productiva fue calculado dividiendo el

Cuadro 1. Datos descriptivos de grupos vacunados contra PCV2 y grupo control. Descriptive data of vaccinated groups against PCV2 and control group.

\begin{tabular}{lcccc}
\hline \multirow{2}{*}{ Variable } & \multicolumn{3}{c}{ Animales vacunados \pm DE } & \multirow{2}{*}{ Control } \\
\cline { 2 - 4 } & Cerdas & Lechones & Cerdas y lechones & 18 \\
\hline Número de cerdas & 18 & 20 & 20 & $4,7 \pm 1,4$ \\
Paridad & $4,6 \pm 1,5$ & $4,5 \pm 1,7$ & $4,6 \pm 1,7$ & $10,1 \pm 1,6$ \\
Lechones vivos por camada & $10,2 \pm 1,7$ & $10,6 \pm 1,6$ & $10,7 \pm 1,8$ & 164 \\
Número de lechones (semana 3) & 166 & 200 & 206 & \\
\hline
\end{tabular}

Media \pm desviación estándar (DE). En cada columna indican diferencias significativas $(\mathrm{P}<0,05)$ en comparación con el grupo control. 
número de cerdos que murieron en esa etapa sobre el número de cerdos asignados a ese grupo, multiplicado por 100 . Los cerdos que murieron a lo largo del periodo de estudio que no mostraron signos clínicos del PMWS fueron diagnosticados por inmunohistoquímica (Kim y Chae 2004).

Todos los cerdos fueron pesados individualmente después de nacer (semana 1), al destete (semana 3), al entrar al sitio tres o etapa de finalización (semana 10) y el día previo a su venta (semana 22). Se determinó la ganancia diaria de peso para cada cerdo, para la etapa de lactancia, destete y finalización. La GDP se calculó restando el peso ganado al inicio de cada etapa al peso final de la misma, dividido por el número de días que permanecieron en la etapa productiva. Finalmente se calculó la GDP para cada cerdo desde el día que fue vacunado (semana 3 ) hasta el día previo a su venta (semana 22).

\section{ANÁLISIS ESTADÍSTICO}

Los datos sobre porcentaje de mortalidad y GDP fueron analizados utilizando un análisis de varianza (ANOVA) usando el procedimiento modelo lineal general (GLM), comparando el grupo control contra los grupos de cerdos vacunados para el modelo de efectos fijos, paridad y número de lechones por parto como efectos aleatorios. Se utilizó el programa SPSS versión 17 para Windows (SPSS Inc, Chicago IL, USA). Una $\mathrm{P}<0,05$ fue considerada como significativa.

\section{RESULTADOS}

La media de lechones nacidos vivos por camada para animales vacunados: cerdas, lechones, cerdas y lechones fue de 10,2, 10,6 y 10,7 respectivamente. Así mismo, el número de lechones nacidos vivos en el grupo control fue de 10,1 (cuadro 1). El número de partos promedio para el grupo control fue de 4,7, mientras que para los diferentes grupos de animales vacunados (cerdas, lechones y cerdas y lechones) fueron de 4,6, 4,5 y 4,6 respectivamente. Los diferentes grupos de cerdos inmunizados, no mostraron diferencias significativas en el número de partos en comparación con el grupo control (cuadro 1).

\section{EFECTO DE LA VACUNACIÓN SOBRE LA GANANCIA DIARIA DE PESO}

En la etapa de lactancia (semana 1-3, cuadro 2), la GDP para el grupo control fue de 227,0 g/d, el grupo de cerdas y lechones inmunizados obtuvo la mayor ganancia de peso $(238,6 \mathrm{~g} / \mathrm{d}, \mathrm{P}<0,05)$, mientras que en las cerdas vacunadas se observó una menor ganancia de peso $(232,3 \mathrm{~g} / \mathrm{d}, \mathrm{P}>0,05)$. Para la etapa de destete (semana 3-10), la GDP de los tres grupos evaluados tuvieron un aumento significativo en comparación con el grupo control. Los lechones, cerdas y lechones vacunados, para la etapa de finalización (semana 10-22), la GDP fue significativa $(\mathrm{P}<0,05)$. Los lechones, cerdas y lechones vacunados mostraron un efecto significativo sobre la GDP (699,4 g/d y 704,0 g/d) para el periodo comprendido entre la semana 3 y 22. Se encontraron diferencias significativas $(\mathrm{P}<0,05)$ para los tres grupos de cerdos vacunados para la GDP en las tres etapas evaluadas durante el estudio (semana 1-22, cuadro 2), sobresaliendo el grupo de cerdas y lechones inmunizados $(656,2 \mathrm{~g} / \mathrm{d})$ en comparación a los demás grupos. El grupo de cerdas vacunadas obtuvo el menor incremento en la GDP $(634,0 \mathrm{~g} / \mathrm{d})$.

\section{EFECTO DE LA VACUNACIÓN SOBRE EL PORCENTAJE DE MORTALIDAD}

Se evaluó el efecto de tres diferentes protocolos de vacunación sobre el porcentaje de mortalidad para el periodo de estudio (semana 1-22), el cual fue dividido en tres periodos: lactación (semana 1-3), destete (semana 3-10) y finalización (semana 10-22, cuadro 3). El mayor porcentaje de mortalidad fue en la etapa de destete para el grupo control $(12,20 \%)$, mientras que el menor porcentaje fue para el grupo de cerdas y lechones inmunizados (1,52\%, 10-22 semanas). El grupo de cerdas y lechones vacunados para la etapa de lactación y destete mostraron diferencias significativas en el porcentaje de mortalidad $(3,74 \%$ y $3,33 \%)$ cuando se les comparó con el grupo control $(9,89 \%$ y $12,20 \%, \mathrm{P}<0,05)$. Así mismo, dos grupos de animales vacunados: lechones, $\mathrm{y}$ cerdas y lechones $(2,13 \%$ y $1,52 \%)$, respectivamente,

Cuadro 2. Media de la ganancia diaria de peso $(\mathrm{g} / \mathrm{d})$ durante diferentes periodos de estudio. Average daily weight gain $(\mathrm{g} / \mathrm{d})$ of piglets during different study intervals.

\begin{tabular}{lccccc}
\hline \multirow{2}{*}{$\begin{array}{l}\text { Animales } \\
\text { vacunados }\end{array}$} & \multicolumn{4}{c}{ Etapa, periodo en semanas \pm DE } \\
\cline { 2 - 6 } & Lactancia & Destete & Finalización & $3-22$ & $1-22$ \\
\hline Cerdas & $1-3$ & $3-10$ & $87-22$ & $681,8 \pm 84,7$ & $634,0 \pm 57,4^{\mathrm{a}}$ \\
Lechones & $232,3 \pm 27,5$ & $400,4 \pm 46,8^{\mathrm{a}}$ & $874,9 \pm 108,0$ & $638,8 \pm 71,2^{\mathrm{a}}$ \\
Cerdas y lechones & $238,0 \pm 29,9^{\mathrm{a}}$ & $417,8 \pm 54,3^{\mathrm{a}}$ & $883,9 \pm 116,6^{\mathrm{a}}$ & $699,4 \pm 92,9^{\mathrm{a}}$ & $6,7^{\mathrm{a}}$ \\
Control & $238,6 \pm 29,7^{\mathrm{a}}$ & $423,2 \pm 57,2^{\mathrm{a}}$ & $916,5 \pm 109,7^{\mathrm{a}}$ & $704,0 \pm 89,7^{\mathrm{a}}$ & $656,2 \pm 56,3^{\mathrm{a}}$ \\
\hline
\end{tabular}

Media \pm desviación estándar (DE). Superíndices (a) en cada columna indican diferencias significativas $(\mathrm{P}<0,05)$ en comparación con el grupo control. 
Cuadro 3. Porcentaje de mortalidad (\%) de grupos vacunados contra PCV2 y grupo control. Mortality rates (\%) of vaccinated groups against PCV2 and control group.

\begin{tabular}{|c|c|c|c|c|c|}
\hline \multirow[b]{2}{*}{ Animales vacunados } & \multicolumn{5}{|c|}{ Etapa, periodo en semanas $\pm \mathrm{DE}$} \\
\hline & $\begin{array}{c}\text { Lactancia } \\
1-3\end{array}$ & $\begin{array}{c}\text { Destete } \\
3-10\end{array}$ & $\begin{array}{c}\text { Finalización } 1 \\
0-22\end{array}$ & $3-22$ & $1-22$ \\
\hline Cerdas & $9,78 \pm 0,06$ & $9,64 \pm 0,10$ & $3,33 \pm 0,06$ & $12,97 \pm 0,12$ & $22,75 \pm 0,11$ \\
\hline Lechones & $5,66 \pm 0,06$ & $6,00 \pm 0,07$ & $2,13 \pm 0,03^{\mathrm{a}}$ & $8,13 \pm 0,06^{\mathrm{a}}$ & $13,79 \pm 0,09$ \\
\hline Cerdas y lechones & $3,74 \pm 0,05^{\mathrm{a}}$ & $3,33 \pm 0,05^{\mathrm{a}}$ & $1,52 \pm 0,03^{\mathrm{a}}$ & $5,40 \pm 0,05^{\mathrm{a}}$ & $9,14 \pm 0,07^{\mathrm{a}}$ \\
\hline Control & $9,89 \pm 0,08$ & $12,20 \pm 0,10$ & $5,56 \pm 0,07$ & $17,75 \pm 0,09$ & $27,64 \pm 0,10$ \\
\hline
\end{tabular}

Media \pm desviación estándar (DE). Superíndices (a) en cada columna indican diferencias significativas $(\mathrm{P}<0,05)$ en comparación con el grupo control.

mostraron reducciones significativas en el porcentaje de mortalidad $(\mathrm{P}<0,05)$ en la etapa de finalización, cuando se les comparó con el grupo control (5,56\%). Las cerdas y lechones vacunados mostraron un efecto significativo sobre el porcentaje de mortalidad $(5,40 \%, \mathrm{P}<0,05)$ para el periodo posterior a la inmunización (semana 3-22). El porcentaje de mortalidad acumulada para el periodo de estudio (semana 1-22) en el grupo control fue de $27,64 \%$, las cerdas y lechones vacunados mostraron diferencias significativas $(9,14 \%, \mathrm{P}<0,05)$; mientras que el grupo de cerdas vacunadas y el grupo de lechones inmunizados no se observaron diferencias significativas $(22,75 \%$ y $13,79 \%, P>0,05)$.

\section{DISCUSIÓN}

En México existen varias vacunas comerciales contra PCV2, incluyendo una vacuna inactivada con adyuvante usada en cerdas para inducir una respuesta inmune celular que es transferida pasivamente después de la ingestión de calostro a los lechones, los cuales aumentan significativamente el número de células secretoras de IFN- $\gamma$, incrementando la respuesta de hipersensibilidad retardada, induciendo una fuerte respuesta proliferativa de células mononucleares de sangre periférica (Oh y col 2012). Los lechones son vacunados a las 3 semanas de edad, cuando los anticuerpos maternales descienden. La vacuna induce una respuesta de anticuerpos neutralizantes, reduciendo o retrasando la infección por PCV2 durante el destete o engorda (Fort y col 2008).

En este estudio, el grupo de lechones, cerdas y lechones inmunizados mostraron GDP significativas para las dos etapas (destete y finalización) posteriores a la aplicación de la vacuna Circovac ${ }^{\circledR}$. La GDP para los lechones desde la administración de la vacuna hasta la salida al mercado (semana 3-22) fue de 699,4 g/d y para el grupo control 680,7 g/d. Así mismo, la GDP para los lechones en la etapa de finalización o engorda (semana 10-22) fue de 883,9 g/d y para el grupo control 860,4 g/d. Heissenberger y col (2010) demostraron que lechones vacunados

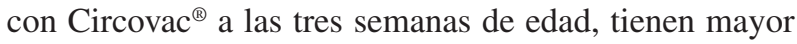
$\operatorname{GDP}(584,8 \mathrm{~g})$, frente al grupo control $(519,9 \mathrm{~g})$ en el periodo de 13-21 semanas; la principal viremia se presenta en las semanas 13-28 cuando la GDP es más evidente (651,04 g vs 609,61 g). Soukup y Dušek 2010 estudiaron el efecto de la vacunación de lechones con $\operatorname{Circovac}^{\circledR}$ a los 21 días de edad en granjas con problemas de PCVD, mostrando GDP de $434 \mathrm{~g}$ durante las etapas posteriores al destete, así como en la etapa de engorda $752 \mathrm{~g}$; los controles para ambas etapas mostraron GDP de $359 \mathrm{~g}$ y $766 \mathrm{~g}$ respectivamente. Cerdas y lechones vacunados con Porcilis ${ }^{\circledR}$ (Intervet) mostraron GDP menores a los observados en el presente estudio (626,0 g vs 704,0 g) en periodos semejantes (4-25 semanas vs 3-22 semanas); así mismo, hembras inmunizadas contra PCV2 (612,0 g vs 681,8 g) (Fraile y col 2012).

En nuestro estudio, se observó una reducción del $6,20 \%$ en mortalidad para el grupo de lechones vacunados (semana 3-10) en comparación con el grupo control. En Austria, se utilizaron 1.105 lechones divididos en dos grupos para ser inmunizados con $0,5 \mathrm{ml}$ de Circovac $^{\circledR}$ a las 3 semanas de edad, observándose una reducción en el porcentaje de mortalidad del 5,15\% para las semanas 13-21, que corresponden al periodo principal de viremia; para las semanas 13-28 se observó una reducción significativa del 7,4\% en la mortalidad de los lechones (Heissenberger y col 2010). Por otro lado, los lechones inmunizados, mostraron una reducción significativa en el porcentaje de mortalidad para el periodo posterior a la vacunación (semana 3-22) en comparación con el grupo control ( $8,13 \%$ vs $17,75 \%)$, así como para el periodo de finalización (semana 10-22, 2,13\% vs 5,56\%). Estos resultados concuerdan con los obtenidos al vacunar lechones con Circovac $^{\circledast}$ a los 21 días de edad, los cuales mostraron una reducción en el porcentaje de mortalidad en comparación con el grupo control (3,68 vs 13,35\%), mientras que para la etapa de engorda fue de 4,32 vs $6,46 \%$ (Soukup y col 2010). Cerdas y/o lechones vacunados con Porcilis ${ }^{\circledR}$ (Intervet) no mostraron diferencias significativas en el porcentaje de mortalidad cuando se les comparó con el grupo control para la etapa de destete a finalización (Fraile y col 2012).

Pejsak y col (2010) utilizaron diferentes protocolos de inmunización para tres grupos de animales: cerdas, lechones y por último cerdas y lechones. Las cerdas fueron vacunadas con $\operatorname{Circovac}^{\circledR}$ en dos ocasiones con un inter- 
valo de 4 semanas antes del parto, los lechones fueron inmunizados a la edad de 4 semanas, mientras que en el tercer grupo las cerdas fueron vacunadas en dos ocasiones con un intervalo de 4 semanas y los lechones en la semana 4 y 7 de edad. Los porcentajes de mortalidad y GDP para los distintos grupos fueron: cerdas $(16,93 \%$ y $635,2 \mathrm{~g})$, lechones $(16,12 \%$ y $640,3 \mathrm{~g})$, y cerdas y lechones $(15,35 \%$ y $656,0 \mathrm{~g})$ y durante la presentación del PMWS (28,76\% y 568,5 g). Estos datos coinciden con la GDP observada en nuestro estudio; sin embargo, el porcentaje de mortalidad en los diferentes grupos vacunados para el periodo de estudio fueron diferentes: cerdas $(22,75 \%$ y $634,0 \mathrm{~g})$, lechones $(13,79 \%$ y $638,8 \mathrm{~g})$, cerdas y lechones $(9,14 \%$ y $656,2 \mathrm{~g})$ y para el grupo control (27,64\% y $618,2 \mathrm{~g})$.

En conclusión, este estudio demuestra que la vacunación de cerdas y lechones con $\operatorname{Circovac}^{\circledR}$ reduce significativamente el porcentaje de mortalidad de cerdos inmunizados, así como un aumento en la ganancia diaria de peso en comparación con el grupo de animales sin vacunar. Estos datos sugieren que la vacunación contra PCV2 puede ser útil en el control de la PCVD, influyendo en los parámetros productivos y reduciendo las pérdidas económicas asociadas a la circovirosis porcina.

\section{REFERENCIAS}

Beach NM, XJ Meng. 2012. Efficacy and future prospects of commercially available and experimental vaccines against porcine circovirus type 2 (PCV2). Virus Res 164, 33-42.

Calsamiglia M, L Fraile, A Espinal, A Cuxart, C Seminati, M Martín, E Mateu, M Domingo, J Segalés. 2007. Sow porcine circovirus type 2 (PCV2) status effect on litter mortality in postweaning multisystemic wasting syndrome (PMWS). Res Vet Sci 82, 299-304.

Chae C. 2012. Commercial porcine circovirus type 2 vaccines: Efficacy and clinical application. Vet J 194, 151-157.

Fort M, M Sibila, A Allepuz, E Mateu, F Roerink, J Segalés. 2008. Porcine circovirus type 2 (PCV2) vaccination of conventional pigs prevents viremia against PCV2 isolates of different genotypes and geographic origins. Vaccine 26, 1063-1071.

Fraile L, M Sibila, M Nofrarías, R López-Jimenez, E Huerta, A Llorens, S López-Soria, D Pérez, J Segalés. 2012. Effect of sow and piglet porcine circovirus type 2 (PCV2) vaccination on piglet mortality, viraemia, antibody titre and production parameters. Vet Microbiol 161, 229-234.

Gerber PF, FM Garrocho, AM Lana, ZI Lobato. 2011. Serum antibodies and shedding of infectious porcine circovirus 2 into colostrum and milk of vaccinated and unvaccinated naturally infected sows. Vet $J$ 188, 240-242.

Harding J. 1998. Postweaning multisystemic wasting syndrome: epidemiology and clinical presentation. J Swine Health Prod 6, 249-254.

Harding JC. 2004. The clinical expression and emergence of porcine circovirus 2. Vet Microbiol 98, 131-135.

Heissenberger B, A Ladinig, C Lang, C Püschel, M Ritzmann.

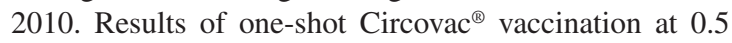

$\mathrm{ml}$ in piglets on weight and mortality parameters. Proceedings of the $21^{\text {st }}$ International Pig Veterinary Society Congress, Vancouver, Canada, Pp 329.

Kekarainen T, K McCullough, M Fort, C Fossum, J Segalés, GM Allan. 2010. Immune responses and vaccine-induced immunity against Porcine circovirus type 2. Vet Immunol Immunopathol 15, 185-193.

Kim J, C Chae. 2004. A comparison of virus isolation, polymerase chain reaction, immunohistochemistry, and in situ hybridization for the detection of porcine circovirus 2 and porcine parvovirus in experimentally and naturally coinfected pigs. $J$ Vet Diagn Invest 16, 45-50.

Kurmann J, T Sydler, E Brugnera, E Buergi, M Haessig, M Suter, X Sidler. 2011. Vaccination of dams increases antibody titer and improves growth parameters in finisher pigs subclinically infected with porcine circovirus type 2. Clin Vaccine Immunol 18, 1644-1649.

Mikami O, H Nakajima, K Kawashima, M Yoshii, Y Nakajima. 2005. Nonsuppurative myocarditis caused by porcine circovirus type 2 in a weak-born piglet. J Vet Med Sci 67, 735-738.

Oh Y, HW Seo, K Han, C Park, C Chae. 2012. Protective effect of the maternally derived porcine circovirus type 2 (PCV2)-specific cellular immune response in piglets by dam vaccination against PCV2 challenge. J Gen Virol 93, 1556-1562.

Opriessnig T, AR Patterson, DM Madson, N Pal, S Ramamoorthy, XJ Meng, PG Halbur. 2010. Comparison of the effectiveness of passive (dam) versus active (piglet) immunization against porcine circovirus type 2 (PCV2) and impact of passively derived PCV2 vaccine-induced immunity on vaccination. Vet Microbiol 142, 177-183.

Pejsak Z, K Podgórska, M Truszczyński, P Karbowiak, T Stadejek. 2010. Efficacy of different protocols of vaccination against porcine circovirus type 2 (PCV2) in a farm affected by postweaning multisystemic wasting syndrome (PMWS). Comp Immunol Microbiol Infect Dis 33, e1-e5.

Rodríguez-Arrioja GM, J Segalés, M Calsamiglia, AR Resendes, M Balasch, J Plana-Duran, J Casal, M Domingo. 2002. Dynamics of porcine circovirus type 2 infection in a herd of pigs with postweaning multisystemic wasting syndrome. Am J Vet Res 63, 354-357.

Segalés J, M Domingo. 2002. Postweaning multisystemic wasting syndrome (PMWS) in pigs. A review. Vet $Q 24,109$ 124.

Sibila M, M Calsamiglia, J Segalés, P Blanchard, L Badiella, M Le Dimna, A Jestin, M Domingo. 2004. Use of a polymerase chain reaction assay and an ELISA to monitor porcine circovirus type 2 infection in pigs from farms with and without postweaning multisystemic wasting syndrome. Am J Vet Res 65, 88-92.

Soukup I, P Dušek. 2010. Effect of piglets PCV2 vaccination with CIRCOVAC $^{\circledR}$ in the farm with severe porcine circovirus type 2 disease (PCVD) in post-weaning. Proceedings of the $21^{\text {st }}$ International Pig Veterinary Society Congress, Vancouver, Canada, Pp 381.

West KH, JM Bystrom, C Wojnarowicz, N Shantz, M Jacobson, GM Allan, DM Haines, EG Clark, S Krakowka, F McNeilly, C Konoby, K Martin, JA Ellis. 1999. Myocarditis and abortion associated with intrauterine infection of sows with porcine circovirus 2. J Vet Diagn Invest 11, 530-532. 
